\title{
Generalized perfect domination in graphs
}

\author{
B. Chaluvaraju $\cdot$ K.A. Vidya
}

Published online: 26 June 2012

(C) Springer Science+Business Media, LLC 2012

\begin{abstract}
Let $k$ be a positive integer and $G=(V, E)$ be a graph. A vertex subset $D$ of a graph $G$ is called a perfect $k$-dominating set of $G$, if every vertex $v$ of $G$, not in $D$, is adjacent to exactly $k$ vertices of $D$. The minimum cardinality of a perfect $k$-dominating set of $G$ is the perfect $k$-domination number $\gamma_{k p}(G)$. In this paper, we give characterizations of graphs for which $\gamma_{k p}(G)=\gamma(G)+k-2$ and prove that the perfect $k$-domination problem is NP-complete even when restricted to bipartite graphs and chordal graphs. Also, by using dynamic programming techniques, we obtain an algorithm to determine the perfect $k$-domination number of trees.
\end{abstract}

Keywords $k$-Domination $\cdot$ Perfect $k$-domination $\cdot$ NP-Completeness $\cdot$ Dynamic programming

\section{Introduction}

Let $G=(V, E)$ be a finite, undirected and simple graph with $n=|V|$ and $m=|E|$. For $G$, let $\Delta$ denotes the maximum degree and an open neighborhood $N(v)$ be the vertices adjacent to vertex $v$. Let $N[v]=N(v) \cup\{v\}$ be the closed neighborhood of $v$ and $\langle D\rangle$ be the subgraph induced by $D$, where $D \subset V$. A dominating set is a set of vertices $D$ where every vertex of $G$ is in $N[v]$ for some $v \in D$. The domination number $\gamma(G)$ is the minimum order of a dominating set. For review of domination and its related parameters we refer to Acharya et al. (1979) and Haynes et al. (1998a, 1998b).

B. Chaluvaraju $\cdot$ K.A. Vidya $(\bowtie)$

Department of Mathematics, Bangalore University, Central College Campus, Bangalore 560 001, India

e-mail: vidya.mnj@gmail.com

B. Chaluvaraju

e-mail: bchaluvaraju@gmail.com 
For a positive integer $k$, a vertex subset $D$ of a graph $G$ is called a $k$-dominating set of $G$, if any vertex $v$ of $G$ not in $D$ is adjacent to at least $k$ elements of $D$. This class of graph has been studied in Fink and Jacobson (1985). Perfect domination was introduced by Cockayne et al. (1993). For more details we refer to Dejter and Pujol (1995), Fellows and Hoover (1991), Yen and Lee (1996).

In this paper, we continue the study of perfect $k$-domination in graphs which was introduced by Chaluvaraju et al. (2010) and Chaluvaraju and Vidya (2012). For a positive integer $k$, a vertex subset $D$ of a graph $G$ is called a perfect $k$-dominating set of $G$, abbreviated $\mathrm{P} k \mathrm{D}$-set, if any vertex $v$ of $G$ not in $D$ is adjacent to exactly $k$ elements of $D$. The minimum cardinality of a $\mathrm{P} k \mathrm{D}$-set of $G$ is the perfect $k$-domination number, $\gamma_{k p}(G)$. Note that every nontrivial graph $G$ has a $\mathrm{P} k \mathrm{D}$-set, since $V(G)$ is such a set. For graph-theoretical terminology and notation not defined here we follow Harary (1969).

Also, we posed the following application to perfect $k$-domination provided by a specialist giving radiation (or some powerful drug) to a patient. In order to be effective there must be precisely $k$ units administered to the neighboring cells (any more is very dangerous). The cells where the drug is given directly are, unfortunately, weakened or harmed and thus we wish to minimize the number of spots/cells where it is given. Thus we would want a minimum $\mathrm{P} k \mathrm{D}$-set.

Another application can be in the field of networking where we have to install a software to some nodes in a network and other nodes should be exactly adjacent to $k$ nodes where the software is installed, otherwise it causes disturbances in the network. To minimize the cost of installation, we need a minimum $\mathrm{P} k \mathrm{D}$-set.

Motivated by such applications we continued our studies on the parameter.

\section{Characterizations of graphs for which $\gamma_{k p}(G)=\gamma(G)+k-2$}

Theorem 1 (Fink and Jacobson 1985) Let $G$ be a graph with $2 \leq k \leq \Delta(G)$. Then

$$
\gamma_{k}(G) \geq \gamma(G)+k-2 \text {. }
$$

Since $\gamma_{k p}(G) \geq \gamma_{k}(G)$, by Theorem $1, \gamma_{k p}(G) \geq \gamma(G)+k-2$. But this bound is sharp and we have many cases where this bound is attained.

Next theorem gives some interesting characterizations of graphs for which $\gamma_{k p}(G)=\gamma(G)+k-2$, which is the best possible lower bound of the parameter.

Theorem 2 Let $D$ be a PkD-set of $G$. If $\gamma_{k p}(G)=\gamma(G)+k-2$, then the following conditions hold:

(i) $\Delta(\langle D\rangle) \leq k-2$.

(ii) There exist at least two independent vertices $u$ and $v$ in $V-D$ with $N(u) \cap D=$ $N(v) \cap D$.

(iii) Every vertex in $D$ has at least one neighbor in $V-D$.

(iv) Every vertex of $G$ lies on an induced cycle of length 4.

Proof Let $D$ be a P $k$ D-set of $G$. If $\gamma_{k p}(G)=\gamma(G)+k-2$, then 
(i) Let $x \in D$ and if possible $x$ be adjacent to at least $k-1$ vertices in $D$. Then let $S \subset N(x) \cap D$ and $|S|=k-1$. Then each element in $V-D$ is adjacent to at least one element in $D-S$ and each element in $S$ is adjacent to $x$. Thus $D-S$ is a dominating set with $\gamma(G)=\gamma_{k p}(G)-(k-1)=\gamma_{k p}(G)-k+1$, which is a contradiction. Therefore $\Delta(\langle D\rangle) \leq k-2$.

(ii) Let $u \in V-D$. Let $S=N(u) \cap D$. Then $|S|=k$. If possible suppose that for every vertex $v \in V-D, N(v) \cap D \neq S$. Since each vertex in $V-D$ is adjacent to exactly $k$ vertices in $D$, each vertex will be adjacent to at least one vertex in $D-S$. Also every vertex in $S$ is adjacent to $u$. Thus $(D-S) \cup\{u\}$ is a dominating set of cardinality $\gamma_{k p}(G)-k+1$, which is a contradiction. Thus there exist at least one vertex $v$ in $V-D$, such that $N(v) \cap D=S$. Also if all the vertices in $V-D$ having this property are adjacent to each other, then $(D-S) \cup\{u\}$ will be a dominating set which is a contradiction. Thus there exists $u$ and $v$ which are independent.

(iii) Let $u \in D$ and $u$ does not have a neighbor in $V-D$. Let $x \in V-D$ and $S=$ $N(x) \cap D$. Then $|S|=k$. If $v \in S$, then we have the following cases to consider.

Case 1. Suppose $N(u) \cap N(t) \cap D=\phi$ for every $t \in V-D$.

Claim. $D^{\prime}=(D-(S \cup N(u))) \cup\{x, v\}$ is a dominating set of $G$.

Let $t \in V-D$. Then since $t$ is adjacent to $k$ vertices in $D, t$ is adjacent to at least one vertex in $(V-S) \cup\{v\}$ and not to $N(u)$. If $t \in S-\{v\}$, it is dominated by $x$. If $t \in N(u), t$ is dominated by $u$. Hence $D^{\prime}$ is a dominating set of cardinality $\gamma_{k p}-(k+|N(u)|)+2$. Since $N(u)$ has at least one vertex, $\gamma_{k p}-(k+|N(u)|)+$ $2 \leq \gamma_{k p}-k+1$, which is a contradiction.

Case 2. Suppose $N(u) \cap N(t) \neq \phi$.

Claim. $D^{\prime}=(D-(S \cup u)) \cup\{x, v\}$ is a dominating set of $\mathrm{G}$, where $v \in S \cap N(u)$. Let $t \in V-D$. Then by the same argument as above, $t$ is adjacent to at least one vertex in $(V-S) \cup\{v\}$. As in the previous case, if $t \in S-\{v\}, t$ is dominated by $x$ and $u$ is dominated by $v$. Thus $(D-(S \cup\{u\}) \cup\{x, v\}$ is a dominating set where $v \in S \cap N(u)$. This also leads to a contradiction. Thus $u$ has at least one neighbor in $V-D$.

(iv) We have two cases to consider:

Case 1. Suppose $x \in V-D$. Let $S=N(x) \cap D$ and $X=\{y \in V-D / N(y) \cap$ $D=S$. By Theorem 2.2(ii), $X$ is not empty and there exists $y \in X$ such that $x$ and $y$ are not adjacent. Also by Theorem 2.2(i), $\Delta(\langle D\rangle) \leq k-2$. Since $S$ is of cardinality $k$, there exist two vertices $a, b \in S$ such that $a$ and $b$ are independent. Then $\{x, a, y, b\}$ forms a cycle of length 4 .

Case 2. Suppose $x \in D$. Then by Theorem 2.2(iii), there exists a vertex $u \in V-$ $D$ such that $u$ is adjacent to $x$. Let $S=N(u) \cap D$. Then again by Theorem 2.2(ii), there exists $v \in V-D$ such that $N(v) \cap D=S$ and $u$ and $v$ are independent. Also by the same argument as in case 1 , we have $a, b \in S$ such that $a$ and $b$ are independent. Then $\{u, a, v, b\}$ forms a cycle of length 4 . 
Corollary 3 If there exists a vertex $x$, which is not contained in any induced cycle of length 4 then $\gamma_{k p}(G) \geq \gamma(G)+k-1$.

Corollary 4 If $G$ is a triangulated graph, then $\gamma_{k p}(G) \geq \gamma(G)+k-1$.

Corollary 5 For any graph $G, \gamma_{k p}(G)=\gamma(G)$ only if $k=2$ and in such case the perfect 2-dominating set is an independent set.

\section{Complexity results}

In Chaluvaraju et al. (2010) we studied the complexity of P $k \mathrm{D}$-set in general graphs.

Theorem 6 (Chaluvaraju et al. 2010) The perfect $k$-dominating set is NP-complete.

In this paper, we study the complexity of the perfect $k$-domination when restricted to some subclasses. Here we prove that the problem is NP-complete even when restricted to bipartite graphs and chordal graphs. For studying complexity we refer to Garey and Johnson (1979).

Instance: A bipartite graph $G$ and a positive integer $p$.

Question: Does $G$ have a perfect $k$-dominating set of cardinality $p$ or less?

Theorem 7 The perfect $k$-dominating set is NP-complete even when restricted to bipartite graphs.

Proof The perfect $k$-dominating set is in NP. If given a set $S,|S|<p$, the neighborhoods of all vertices $v \in(V-S)$ could be checked in polynomial time to ensure that $|N(v) \cap S|=k$.

Let $X=\left\{x_{1}, x_{2}, \ldots, x_{3 q}\right\}$ and $C=\left\{C_{1}, C_{2}, \ldots, C_{t}\right\}$ be an arbitrary instance of $X 3 C$, where $|X|=3 q,|C|=t$ and $p=t+2(k-1)$. The construction of a graph $G$ is given by creating a vertex $x_{i}$ for each element $x_{i} \in X$ and a component consisting of a path $P_{2}$ with vertices labeled $c_{j}$ and $s_{j}$ for every subset $C_{j} \in C$. Add new vertices $U=\left\{u_{1}, u_{2}, \ldots, u_{k-1}\right\}$ adjacent to all $s_{j}$ 's and $x_{j}$ 's. Also add new vertices $V=$ $\left\{v_{1}, v_{2}, \ldots, v_{k-1}\right\}$ adjacent to all $c_{j}$ 's. Also add the edges $E^{\prime}=\left\{x_{i} c_{j}: x_{i} \in C_{j}\right\}$. Let $S^{\prime}=\left\{s_{1}, s_{2}, \ldots, s_{t}\right\}$. From the Fig. 1 it is clear that the graph constructed is a bipartite graph.

Suppose $C^{\prime}$ is an exact cover of $C$. Then $S=\left\{c_{j}: C_{j} \in C^{\prime}\right\} \cup\left\{s_{j}: C_{j} \notin C^{\prime}\right\} \cup$ $\left\{u_{1}, u_{2}, \ldots, u_{k-1}\right\} \cup\left\{v_{1}, v_{2}, \ldots, v_{k-1}\right\}$ is a $\mathrm{P} k \mathrm{D}$-set for $G$ with $|S|=t+2(k-1)$.

Now suppose that $S$ such that $|S| \leq t+2(k-1)$ is a P $k$ D-set for $G$. In graph $G$ the degree of $s_{j}$ is $k$. So either $s_{j} \in S$ or all the neighbors of $s_{j}$ are in $S$.

Claim. $S^{\prime} \nsubseteq S$. If possible suppose $S^{\prime} \subseteq S$. Then we have two cases.

Case 1. $U \nsubseteq \subseteq S$. In this case $u_{i}$ has to be adjacent to exactly $k$ vertices. If $t=k$, then perfectness conditions of the $x_{i}$ 's will not be satisfied. If $t<k, u_{i}$ 's can be made 
Fig. 1 NP-Completeness construction for bipartite graph

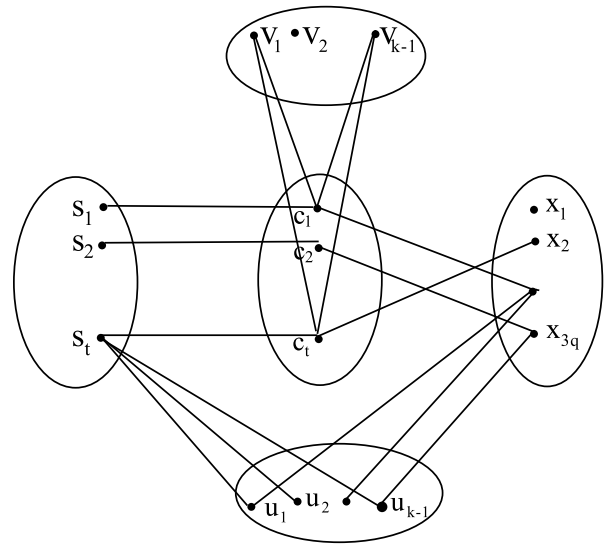

adjacent to exactly $k$ vertices by including some vertices from $X$. Since the $v_{i}$ 's are adjacent only to $C, V \subset S$. Then for the $c_{i}$ 's to be exactly $k$-dominated, no vertex in $X$ should be in $S$, which is a contradiction. If $t>k$, then $U \subset S$ and $V \subset S$, which is a contradiction.

Case 2. $U \subset S$. In this case the $x_{i}$ 's has to be adjacent to exactly one vertex in $C$. Here also $V \subset S$. Then $|S|=\left|S^{\prime}\right|+|U|+|V|+\left|C^{\prime}\right|>2(k-1)+t$, which is a contradiction. Therefore $S^{\prime} \nsubseteq S$.

Thus $U \subset S$ and the $x_{i}$ 's should have exactly one neighbor in $C$, which forms an exact cover for $C$. Therefore $C^{\prime}=\left\{C_{j}: c_{j} \in S\right\}$ is an exact cover for $C$.

Theorem 8 The perfect $k$-dominating set is NP-complete even when restricted to chordal graphs.

Proof The perfect $k$-dominating set is in NP. If given a set $S,|S|<p$ as a witness to yes instance, the neighborhoods of all vertices $v \in(V-S)$ could be checked in polynomial time to ensure that $|N(v) \cap S|=k$.

Let $X=\left\{x_{1}, x_{2}, \ldots, x_{3 q}\right\}$ and $C=\left\{C_{1}, C_{2}, \ldots, C_{t}\right\}$ be an arbitrary instance of $X 3 C$, where $|X|=3 q,|C|=t$ and $p=q+3 q(k-1)$. The construction of a graph $G$ is given by creating a vertex $x_{i}$ for each element $x_{i} \in X$ and a vertex $c_{j}$ for every subset $C_{j} \in C$. Also add new edges with $c_{j}$ 's as end vertices to make $C$ a complete graph. Since $C$ is a complete graph it is clear from the Fig. 2 that the constructed graph is chordal as there are no induced cycles of length more than 3 .

Suppose $C^{\prime}$ is an exact cover of $C$. Then $S=\left\{c_{j}: C_{j} \in C^{\prime}\right\} \cup\left\{u_{1}^{i}, u_{2}^{i}, \ldots, u_{k-1}^{i}\right\}$, $i=1,2, \ldots, 3 q$ is a $\mathrm{P} k \mathrm{D}$-set for $G$ with $|S|=q+3 q(k-1)$.

Now suppose that $S,|S| \leq q+3 q(k-1)$ is a $\mathrm{P} k \mathrm{D}$-set for $G$. Since the degree of $u_{i}^{j}$ is 1 , all the $u_{i}^{j}$ 's has to be included in $S$. Then to satisfy the perfectness condition of $x_{i}$ 's, each $x_{i}$ should have exactly one neighbor in $C$, which forms an exact cover for $C$. Therefore $C^{\prime}=\left\{C_{j}: c_{j} \in S\right\}$ is an exact cover for $C$. 
Fig. 2 NP-Completeness construction for chordal graph

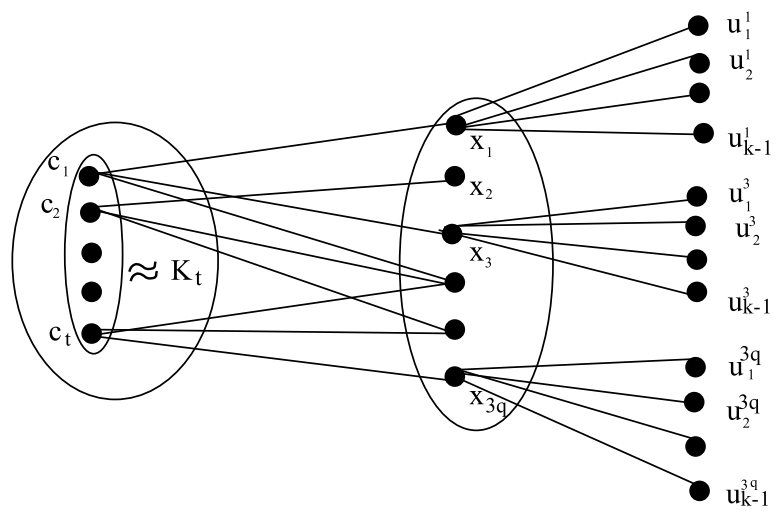

\section{Dynamic programming method for perfect $k$-domination in trees}

Though the problem of finding a P $k \mathrm{D}$-set is NP-complete for general graphs bipartite graphs and chordal graphs, it is possible to find polynomial time algorithms for finding perfect $k$-domination number in some subclasses. As an initiative to this study, we take the subclass, trees.

Dynamic programming is used as a powerful method for solving many discrete optimization problems. Here we develop an algorithm for finding the perfect $k$ domination number in trees by using dynamic programming method, see Chang (1998) and Nemhauser (1966).

Consider a vertex $u \in G$. A minimum $\mathrm{P} k \mathrm{D}$-set, $D$ of $G$ contains or does not contains $u$. So we define the following problems:

$$
\begin{aligned}
& \gamma_{k p}^{1}(G, u)=\min \{|D|: D \text { is a PkD-set of } G \text { and } u \in D\} \\
& \gamma_{k p}^{0}(G, u)=\min \{|D|: D \text { is a P } k \text { D-set of } G \text { and } u \notin D\}
\end{aligned}
$$

Thus we have the following lemma

Lemma 9 Let $G$ be a graph with a specific vertex $u$. Then

$$
\gamma_{k p}(G)=\min \left\{\gamma_{k p}^{1}(G, u), \gamma_{k p}^{0}(G, u)\right\} .
$$

To find the perfect $k$-domination number of the given tree, we start with one vertex of the tree and add the vertices one by one in such a way that at each stage we find the values of $\gamma_{k p}^{1}$ and $\gamma_{k p}^{0}$ of the subgraph formed with the newly added vertex as the $\operatorname{root}($ specific) vertex. For finding these values we use the following concept.

Let $G$ be a graph with specific vertex $u$ and $H$ with specific vertex $v$. Let $I$ be the graph with the specific vertex $u$, which is obtained from the disjoint union of $G$ and $H$ by joining a new edge $u v$. For finding a minimum $\mathrm{P} k \mathrm{D}$-set of $I$, using the above lemma, first we have to find $\gamma_{k p}^{1}(I, u)$ and $\gamma_{k p}^{0}(I, u)$. For this we make use of $\gamma_{k p}^{1}(G, u), \gamma_{k p}^{0}(G, u), \gamma_{k p}^{1}(H, v)$ and $\gamma_{p}^{0}(H, v)$. 
Suppose $D$ is a $\mathrm{P} k \mathrm{D}$-set of $I$ with $u \in D$. Then $D=D^{\prime} \cup D^{\prime \prime}$, where $D^{\prime}$ is a P $k \mathrm{D}$ set of $G$ with $u \in D^{\prime}$ and $D^{\prime \prime}$ is a $\mathrm{P} k \mathrm{D}$-set of $H$ with $v \in D^{\prime \prime}$ or $D^{\prime \prime}$ is a $\mathrm{P} k \mathrm{D}$-set of $H-\{v\}$ with $|N(v) \cap D|=k-1$. For the latter case, we define the following:

$$
\gamma_{k p}^{00}(G, u)=\min \{|D|: D \text { is a PkD-set of }(G-\{u\}) \text { and } N(u) \cap D=k-1\} .
$$

Note that due to the perfectness condition, $\gamma_{k p}^{00}(G, u)$ and $\gamma_{k p}^{0}(G, u)$ do not exist always but $\gamma_{k p}^{1}(G, u)$ exists, since $V(G)$ is a $\mathrm{P} k \mathrm{D}$-set which contains $u$. Whenever $\gamma_{k p}^{0}(G, u)$ and $\gamma_{k p}^{00}(G, u)$ do not exist, we define, $\gamma_{k p}^{0}(G, u)=\gamma_{k p}^{00}(G, u)=\lambda(\lambda>n)$. Also Initialize $\gamma_{k p}^{1}(G, u)=1$. To support the correctness of the algorithm we present the following theorem.

Theorem 10 Let $G$ be a graph with the specific vertex $u$ and $H$ be a graph with specific vertex $v$. Let $I$ be the graph with specific vertex $u$, which is obtained from the disjoint union of $G$ and $H$ by joining a new edge $u v$. Then

$$
\gamma_{k p}^{1}(I, u)= \begin{cases}\gamma_{k p}^{1}(G, u)+\min \left\{\gamma_{k p}^{1}(H, v), \gamma_{k p}^{00}(H, v)\right\} & \text { if } \gamma_{k p}^{00}(H, v)<\lambda, \\ \gamma_{k p}^{1}(G, u)+\gamma_{k p}^{1}(H, v) & \text { else. }\end{cases}
$$

Proof Suppose $D$ is a $\mathrm{P} k \mathrm{D}$-set of $I$ with $u \in D$. Then we have the following cases:

Case 1. $D=D^{\prime} \cup D^{\prime \prime}$ where $D^{\prime}$ is a $\mathrm{P} k \mathrm{D}$-set of $G$ with $u \in D^{\prime}$ and $D^{\prime \prime}$ is a $\mathrm{P} k \mathrm{D}$-set of $H$ with $v \in D^{\prime \prime}$.

Case 2. $D=D^{\prime} \cup D^{\prime \prime}$ where $D^{\prime}$ is a $\mathrm{P} k \mathrm{D}$-set of $G$ with $u \in D^{\prime}$ and $D^{\prime \prime}$ is a $\mathrm{P} k \mathrm{D}$-set of $H-\{v\}$ with $|N(v) \cap D|=k-1$. Thus for a minimum $\mathrm{P} k \mathrm{D}$, we have to take the minimum of $\gamma_{k p}^{1}(H, v)$ and $\gamma_{k p}^{00}(H, v)$. But if there does not exist a P $k \mathrm{D}$-set of $H-\{v\}$ with $|N(v) \cap D|=k-1$, then $\gamma_{k p}^{00}(H, v)=\lambda$. In this case, we have to choose the minimum $\mathrm{P} k \mathrm{D}$-set of $H$ with $v \in D^{\prime \prime}$. Thus the results follows.

Algorithm: Given a tree, we will label vertices of $T$, such that the tree will be a rooted tree at vertex $v_{1}$ and the labels of the vertices will increase as we go to the upper branches of the tree in a breadth first format (Chaluvaraju and Vidya 2012). For example, a tree has to be labeled in the format as in Fig 3.

Now associate two numbers $v_{i}(s)$ and $v_{i}(e)$, to each vertex $v_{i}$ of degree greater than one, as follows.

(i) $v_{i}(s)=\min \left\{j: v_{i}\right.$ is the parent vertex of $\left.v_{j}\right\}$,

(ii) $v_{i}(e)=\max \left\{j: v_{i}\right.$ is the parent vertex of $\left.v_{j}\right\}$.

Let $L\left(v_{i}\right)$ be a label associated with each vertex. Initialize $\gamma_{k p}^{1}\left(v_{i}\right)=1 ; \gamma_{k p}^{0}\left(v_{i}\right)=$ $\lambda ; \gamma_{k p}^{00}\left(v_{i}\right)=\lambda ; L\left(v_{i}\right)=S$ (where ' $S^{\prime}$ stands for sure to be in $D$ ). Now, from $i=n$ to $i=2$, for each vertex $v_{i}$, we find values of $\gamma_{k p}^{1}\left(v_{i}\right), \gamma_{k p}^{0}\left(v_{i}\right)$ and $\gamma_{k p}^{00}\left(v_{i}\right)$.

If $v_{i}$ is a pendent vertex, the initialized values will not change. If $v_{i}$ is not a pendent vertex, let $a=v_{i}(s)$ and $b=v_{i}(e)$. That is, $v_{i}$ is the parent vertex of vertices $v_{a}, v_{a+1}, \ldots, v_{b}$. 
Fig. 3 A labeled tree

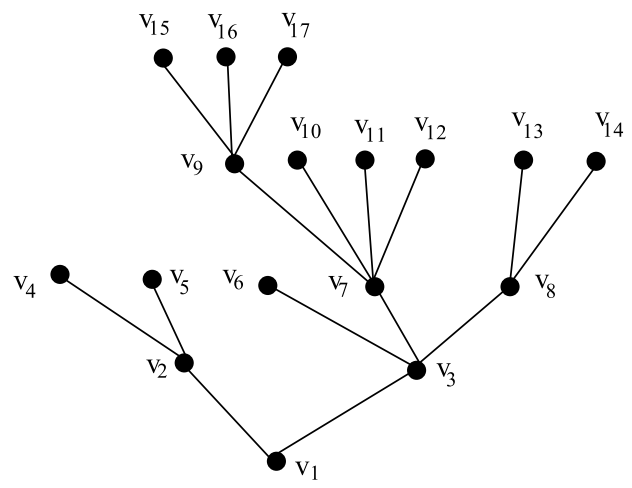

Method to find $\gamma_{k p}^{1}\left(v_{i}\right)$ :

For $t=b$ to $a$

If $\left(\gamma_{k p}^{00}\left(v_{i}\right)<\lambda\right)$

$\gamma_{k p}^{1}\left(v_{i}\right)=\gamma_{k p}^{1}\left(v_{i}\right)+\min \left\{\gamma_{k p}^{1}\left(v_{t}\right), \gamma_{k p}^{00}\left(v_{t}\right)\right\}$

else

$\gamma_{k p}^{1}\left(v_{i}\right)=\gamma_{k p}^{1}\left(v_{i}\right)+\gamma_{k p}^{1}\left(v_{t}\right)$

End if

End for

Thus we get $\gamma_{k p}^{1}\left(v_{i}\right)$.

Correctness of the algorithm: The correctness of the algorithm follows from previous theorem.

Method to find $\gamma_{k p}^{0}\left(v_{i}\right)$ : Let $a$ and $b$ have the same values as above. Then consider the following cases:

Case 1. If $b-a+1<k$, then the vertex $v_{i}$ has only $b-a+1$ child vertices. Thus we cannot find a $\mathrm{P} k \mathrm{D}$-set which does not have $v_{i}$. Then $\gamma_{k p}^{0}\left(v_{i}\right)=\lambda$.

Case 2. If $b-a+1 \geq k$, then let $p$ denotes the number of vertices in the set $C=$ $\left\{v_{a}, v_{a+1}, \ldots, v_{b}\right\}$, with label $S$. Here we have two sub cases.

Subcase 2.1. If $p>k$, then the vertex $v_{i}$ has to be in $\mathrm{P} k \mathrm{D}$-set. Thus $\gamma_{k p}^{0}\left(v_{i}\right)=\lambda$.

Subcase 2.2. If $p \leq k$, we have to include $k-p$ more vertices in $D$, to satisfy the perfectness condition of $v_{i}$. To choose these vertices, we use the following method.

For all the vertices in the set $C$, with label $N$, find $d\left(v_{t}\right)=\gamma_{k p}^{0}\left(v_{t}\right)-\gamma_{k p}^{1}\left(v_{t}\right)$. Then arrange $d\left(v_{t}\right)$ in ascending order and choose first $k-p$ vertices with highest $d\left(v_{t}\right)$ and re-label these vertices as $S$. Thus we have $k$ vertices adjacent to $v_{i}$ which belongs to $D$.

In this case we use the following algorithm to find the value of $\gamma_{k p}^{0}\left(v_{i}\right)$. 
Initialize $\gamma_{k p}^{0}\left(v_{i}\right)=0$

For $t=b$ to $a$

If $L\left(v_{t}\right)=S$

$\gamma_{k p}^{0}\left(v_{i}\right)=\gamma_{k p}^{0}\left(v_{i}\right)+\gamma_{k p}^{1}\left(v_{t}\right)$

else

$\gamma_{k p}^{0}\left(v_{i}\right)=\gamma_{k p}^{0}\left(v_{i}\right)+\gamma_{k p}^{0}\left(v_{t}\right)$

End if

End for

$L\left(v_{i}\right)=N$

Thus we get $\gamma_{k p}^{0}\left(v_{i}\right)$.

Correctness of the algorithm: In this part, our main task was to choose $k$ vertices from set $C$ to include in $D$, so that the cardinality of $D$ is minimum. Since $p$ vertices have label $S$, they are sure to be in $D$. Thus we have to choose $k-p$ vertices from the remaining vertices. For that, we calculate $d\left(v_{t}\right)$ for each of these vertices and a higher value of it indicates that the difference between $\gamma_{k p}^{0}\left(v_{t}\right)$ and $\gamma_{k p}^{1}\left(v_{t}\right)$ is higher. In that case, we include that vertex in $D$ and so $\gamma_{k p}^{1}\left(v_{t}\right)$, which is smaller is added to $\gamma_{k p}^{0}\left(v_{i}\right)$ to ensure the minimality of $\gamma_{k p}^{0}\left(v_{i}\right)$. This clarifies the correctness of the algorithm.

Method to find $\gamma_{k p}^{00}\left(v_{i}\right)$ : In the above method if we replace $k$ by $k-1$, we get $\gamma_{k p}^{00}\left(v_{i}\right)$. [For finding $\gamma_{k p}^{0}\left(v_{i}\right), v_{i}$ should be adjacent to $k$ of its child vertices, while in case of $\gamma_{k p}^{00}\left(v_{i}\right)$, it is $k-1$ of its child vertices.]

For $t=b$ to $a$

If $L\left(v_{i}\right)=S$

$\gamma_{k p}^{00}\left(v_{i}\right)=\gamma_{k p}^{00}\left(v_{i}\right)+\gamma_{k p}^{1}\left(v_{t}\right)$

else

$\gamma_{k p}^{00}\left(v_{i}\right)=\gamma_{k p}^{00}\left(v_{i}\right)+\gamma_{k p}^{0}\left(v_{t}\right)$

End if

End for

Thus we get $\gamma_{k p}^{00}\left(v_{i}\right)$. Based on Lemma 9, $\gamma_{k p}(T)=\min \left\{\gamma_{k p}^{1}\left(v_{1}\right), \gamma_{k p}^{0}\left(v_{1}\right)\right\}$, which gives the perfect $k$-domination number of $T$.

\section{Conclusions}

We have studied some characteristics of graphs for which a lower bound of the parameter is attained. We studied the complexity of the problem in different classes of graphs and proved that the problem is NP-Complete for bipartite and chordal graphs and developed an efficient algorithm for solving the problem in trees. For future research on the topic, we plan to work on other classes of graphs and we propose the following open problems.

Open problem 1 Designing efficient algorithms for other classes of graphs. 
Open problem 2 In general graphs how close can any polynomial algorithm get to the optimal solution?

Acknowledgement We thank referees for their careful reading and their valuable suggestions for improving the paper.

\section{References}

Acharya BD, Walikar HB, Sampathkumar E (1979) Recent developments in the theory of domination in graphs. MRI lecture notes in math. Mehta Research Institute, Allahabad

Chang GJ (1998) Algorithmic aspects of domination in graphs. In: Du D-Z, Pardalos PM (eds) Handbook of combinatorial optimization, vol 3, pp 339-405

Chaluvaraju B, Vidya KA (2012) Bounds on perfect $k$-domination in trees: An algorithmic approach. To appear in Opusc Math 32(4)

Chaluvaraju B, Chellali M, Vidya KA (2010) Perfect $k$-domination in graphs. Australas J Comb 48:175184

Cockayne EJ, Hartnell BL, Hedetniemi ST, Laskar R (1993) Perfect domination in graphs. J Comb Inf Syst Sci 18:136-148

Dejter IJ, Pujol J (1995) Perfect domination and symmetry. Congr Numer 111:18-32

Fellows MR, Hoover MN (1991) Perfect domination. Australas J Comb 3:141-150

Fink JF, Jacobson MS (1985) $n$-Domination in graphs. In: Alavi Y, Schwenk AJ (eds) Graph theory with applications to algorithms and computer science. Wiley, New York, pp 283-300

Garey MR, Johnson DS (1979) Computers and intractability: A guide to the theory of NP-completeness. Freeman, San Francisco

Harary F (1969) Graph theory. Addison-Wesley, Reading, Mass

Haynes TW, Hedetniemi ST, Slater PJ (1998a) Fundamentals of domination in graphs. Marcel Dekker, New York

Haynes TW, Hedetniemi ST, Slater PJ (1998b) Domination in graphs: advanced topics. Marcel Dekker, New York

Nemhauser GL (1966) Introduction to dynamic programming. Wiley, New York

Yen CC, Lee RCT (1996) The weighted perfect domination problem and its variants. Discrete Appl Math $66: 147-160$. 